\title{
A relação entre o desenho da figura humana e a coordenação visomotora em crianças pré-termo aos 6 anos de idade
}

\section{The relationship between human figure drawing and visual-motor coordination in 6-year-old preterm children}

\author{
Daniela Julie Carvalho' ${ }^{1}$ Lívia de Castro Magalhães²
}

\begin{abstract}
CARVALHO, D. J.; MAGALHÃES, L. C. A relação entre o desenho da figura humana e a coordenação visomotora em crianças pré-termo aos 6 anos de idade. Rev. Ter. Ocup. Univ. São Paulo, v. 15, n. 3, p. 98-105, set./dez., 2004.
\end{abstract}

\begin{abstract}
RESUMO: O presente estudo teve como objetivo examinar o desempenho, na idade pré-escolar, de crianças nascidas prematuras em testes perceptuais e visomotores. Como a prematuridade tem impacto no desenvolvimento infantil, é importante avaliar as habilidades percepto-motoras nessa população. Participaram desse estudo 30 crianças, 20 meninas e 10 meninos, com idade gestacional, ao nascimento, $\geq 34$ semanas, recrutados em programa de acompanhamento do desenvolvimento de prematuros. Os instrumentos utilizados para avaliação da amostragem foram os testes de Acuidade Motora (AYRES, 1980), Gestáltico de Bender (KOPPITZ, 1989) e as versões do desenho da Figura humana de Wechsler (1996) e Ayres e Reid (1966). Os resultados mostraram que as crianças pré-termo tiveram desempenho dentro do esperado para a idade, em todos os testes, exceto na acuidade motora ajustada para tempo, o que indica que as crianças nascidas prétermo apresentaram maior lentidão no traçado. Foi encontrada correlação significativa entre as duas versões do Desenho da Figura Humana (Pearson $r=0,76$ ) e entre o peso ao nascimento e a acuidade do traçado (Pearson $r=0,36$ e 0,41 ), porém nenhuma outra correlação entre os testes utilizados atingiu significância. Tais resultados contrastam com a literatura, que indica pior desempenho perceptual e motor em crianças nascidas prematuramente. A pobre correlação entre os escores dos diferentes testes e os sinais de pouca utilidade clínica levam a questionar a validade da instrumentação utilizada. Conclui-se que é essencial o desenvolvimento de instrumentação própria, com normas atualizadas, para crianças brasileiras, que permita maior acuidade dos resultados.
\end{abstract}

DESCRITORES: Prematuro. Desempenho psicomotor. Transtornos das habilidades motoras.

\footnotetext{
${ }^{1}$ Terapeuta Ocupacional, bolsista de Iniciação Científica (PIBIC/CNPq).

${ }^{2}$ Doutora em Psicologia da Educação pela Universidade de Illinois, EUA - Docente do Curso de Terapia Ocupacional - Escola de Educação Física, Fisioterapia e Terapia Ocupacional - Universidade Federal de Minas Gerais, UFMG.

Endereço para correspondência: Daniela Julie Carvalho Becho. Rua José Ede, 95. Centro. CEP: 36200-018, Barbacena, MG. e-mail: danibecho @hotmail.com.
} 
$\mathrm{E}$ studos sobre o desenvolvimento de recémnascidos pré-termo mostram que a sobrevivência dos quadros mais severos de prematuridade vem aumentando, graças aos avanços tecnológicos no cuidado intensivo neonatal (GROOT, 2000). Esses bebês, no entanto, estão sujeitos a apresentar seqüelas neurológicas, o que contribui para manter estáveis as taxas de prevalência de PC, mesmo quando medidas preventivas vêm resultando em redução significativa nos problemas pré e perinatais (SCPE, 2000). Apesar de as seqüelas neurológicas mais severas serem detectadas precocemente, observa-se que muitas crianças apresentam problemas tardios, na idade pré-escolar ou escolar. Dentre as alterações observadas, percebe-se que, a medida que a criança cresce e enfrenta maiores desafios, surgem tanto os distúrbios de aprendizagem e os problemas de comportamento, caracterizados principalmente pelo déficit de atenção, como as falhas perceptuais e motoras, que influenciam a escrita e outras habilidades necessárias para o bom desempenho escolar (CARVALHO et al., 2001; GOYEN et al., 1998; LUOMA; MARTIKAINEM, 1998; MAGALHÃES et al., 1999, 2003; WOLKE; MEYER, 1999).

A relação entre aspectos perceptuais e motores, mas especificamente entre a coordenação visomotora e as dificuldades escolares, é bem discutida na literatura (BERRY, 1997; KEPHART, 1990), por isso é importante avaliar o desempenho percepto-motor em crianças expostas a fatores de risco, como é o caso da prematuridade. Existem vários instrumentos específicos para avaliar a coordenação visomotora, dentre eles, são bastante citados na literatura, o teste Gestáltico Bender (KOPPITZ, 1989) que avalia a percepção viso-espacial da criança por meio da cópia de figuras geométricas, o Teste de Acuidade Motora (AYRES, 1980), que é uma prova de traçado, e o Teste de Integração Viso-Motora (BERRY, 1997), que também examina a integração visomotora por meio de cópia de figuras.

Outro instrumento bastante usado para avaliar a coordenação visomotora e o esquema corporal é o teste do desenho da figura humana. Esse é um teste que apresenta várias versões, as quais são utilizadas de maneira diferente pelos profissional. Goodenough (1926 apud AYRES, 1966) deu início aos estudos na área, ao criar um método para avaliar a inteligência por meio da análise do desenho da figura humana. Com base no trabalho de Goodenough, outros autores desenvolveram instrumentos semelhantes. Koppitz (1989) desenvolveu um sistema de pontuação do desenho bastante usado no Brasil, apesar de não ser estandardizado para nossas crianças. Recentemente,
Wechsler (1996) desenvolveu uma versão brasileira de avaliação cognitiva infantil, centrada no desenho da figura feminina e masculina.

Apesar da ênfase cognitiva na psicologia, pesquisadores de áreas diferentes usam o desenho da figura humana com outros objetivos. Na Terapia Ocupacional, Ayres e Reid (1966) desenvolveram uma versão do teste do desenho da figura humana para avaliar aspectos perceptivos e visomotores. Brittain e Chien (1983) usaram o desenho da Figura humana para observar a habilidade da criança ao nomear as diferentes partes do corpo e construir o corpo de um homem com essas partes. Melo (1997), educador físico, utilizou o desenho da Figura humana associado a atividades físicas, para descrever a construção do esquema corporal em crianças na idade pré-escolar. Vê-se que o teste do desenho da Figura humana é utilizado para estimar tanto a cognição como aspectos perceptivos e visomotores do desenvolvimento infantil. Psicólogos tendem a interpretar o desenho dentro de um enfoque cognitivo ou emocional, já terapeutas ocupacionais e educadores físicos tendem a considerar o desenho da Figura humana como uma representação de habilidades perceptomotoras.

Na Terapia Ocupacional, o trabalho de Ayres e Reid (1966) foi pioneiro no uso do desenho da figura humana com crianças na idade pré-escolar. Esses autores desenvolveram um sistema de pontuação e concluíram que o desenho do auto-retrato pode ser bom indicativo de disfunção percepto-visual. Anos mais tarde, ShotDeGraff e Slansky (1989) demonstraram que o escore do desenho do auto-retrato de Ayres e Reid podia ser usado como indicador precoce de atrasos no desenvolvimento percepto-motor.

Testes percepto-motores, incluindo o desenho da figura humana, também são utilizados para avaliar aspectos de prontidão para alfabetização e predição do desempenho escolar. Em 1983, Oakland e Dowling encontraram uma alta correlação entre o desenho da figura humana e habilidades como a capacidade conceitual, verbal, visomotora e discriminativa, que são necessárias para o bom desempenho escolar. No Brasil, Bandeira e Hutz (1994) avaliaram o rendimento escolar de crianças aos 6 e 7 anos, utilizando o desenho da figura humana, versão de Koppitz, e o teste de Bender. Eles observaram que tais testes, quando interpretados juntos, são úteis para o diagnóstico precoce de dificuldades escolares.

Vê-se que o desenho da figura humana, tomado isoladamente ou associado a outros testes perceptomotores, vem sendo usado para detectar problemas relacionados a dificuldades escolares em crianças. Esses 
mesmos instrumentos também são usados na avaliação do desenvolvimento de crianças nascidas pré-termo (MAGALHÃES, 2003), sendo importante examinar o desempenho de tais crianças nesse tipo de teste.

Neste estudo, examinou-se o desempenho de crianças com história de prematuridade, em dois testes de coordenação visomotora; o Teste Gestático de Bender (KOPPITZ, 1989) e o Teste de Acuidade Motora - MAC - (AYRES, 1980), e em duas versões do Teste do Desenho da Figura Humana, a versão cognitiva de Weschsler (1996) e a versão perceptomotora de Ayres e Reid (1966). Nosso objetivo foi examinar os seguintes aspectos: (a) a relação entre prematuridade e coordenação visomotora; (b) se existe correlação entre os escores dos testes de desenho da figura humana e os testes de coordenação visomotora; (c) se o desempenho das crianças, nas duas versões do teste do desenho da figura humana, é significativamente diferente. A expectativa inicial foi de encontrar correlações significativas entre os testes, incluindo o desenho da figura humana, uma vez que a coordenação visomotora parece ser essencial para o traçado. Apesar do sistema diferente de pontuação, as versões cognitiva e percepto-motora do desenho da figura humana devem apresentar forte correlação, pois têm origem comum. Esperava-se também que um número significativo de crianças pré-termo apresentassem desempenho abaixo da expectativa para a idade, nos quatro testes.

\section{MATERIAISE MÉTODOS}

\section{A - Participantes}

Os participantes deste estudo foram selecionados no banco de dados do Ambulatório da Criança de Risco - ACRIAR da Universidade Federal de Minas Gerais (UFMG), que é um programa de acompanhamento longitudinal do desenvolvimento, até os sete anos de idade, de recém-nascidos pré-termo. Foram incluídas todas as crianças de 6 anos de idade, que nasceram com até 34 semanas de idade gestacional e que tinham desenho da figura humana e escores completos dos testes Bender e de Acuidade Motora. De acordo com esse critério, foram selecionadas 30 crianças, sendo 20 meninas e 10 meninos, com idades que variaram de 6 anos a 6 anos e 11 meses. O ACRIAR, como a maioria dos ambulatórios Universitários, atende predominantemente à população de baixa renda, cujas crianças têm menor acesso a ensino pré-escolar regulamentado.
Tabela 1 - Caracterização da amostragem.

\begin{tabular}{l|l|l}
\hline & Média (desvio padrão) & Amplitude \\
\hline Idade gestacional & $31,33( \pm 2,38)$ semanas & $27-34$ \\
Peso ao nascimento & $1.369,67( \pm 148,48)$ gramas & $660-2.280$ \\
Idade atual & $72,6( \pm 1,94)$ meses & $72-81$ \\
\hline
\end{tabular}

B - Instrumentação

Foram utilizados quatro testes de traçado. O Teste de Acuidade Motora (AYRES, 1980), aqui denominado MAC (Motor Acuraccy Test), examina a coordenação visomotora por meio da medida da acuidade do traçado em cima de uma linha curva, em formato de borboleta. A distância traçada fora da linha é medida com um conta-milhas, e resulta no escore de Acuidade (MAC - Acuidade). A combinação do tempo gasto no traçado e a acuidade resulta em um escore ajustado para o tempo (MAC - Ajustado). Quanto maior a pontuação da criança, melhor sua acuidade motora.

O Teste Gestáltico Bender (KOPPITZ, 1989) foi usado com o objetivo de avaliar a coordenação visomotora e a percepção viso-espacial das crianças. O teste constitui-se na cópia de 9 figuras geométricas, e o escore é dado de acordo com o número de erros cometidos. Quanto menor a pontuação, melhor o desempenho visoespacial. No presente estudo, foram seguidas as normas de aplicação e escore recomendadas por Koppitz (1989) e o estudo brasileiro de Kroeff (1988), estimativa para crianças de escolas públicas, foi utilizado para verificar se o desempenho no teste estava dentro da média esperada para a idade.

O desenho da figura humana foi pontuado de duas formas:

a) Versão de Wechsler (1996), na qual a criança ganha 1 ponto para cada parte do corpo representada no desenho, de acordo com critérios específicos para correção do teste. O resultado bruto é obtido pela soma dos pontos e pode ser convertido em percentil e escore padronizado, o que permite estimar o nível de desempenho da criança em relação à sua faixa etária. Apesar de ser recomendado que a criança faça dois desenhos, um de cada sexo, na rotina de acompanhamento do ACRIAR, devido à limitação de tempo, solicitou-se apenas um desenho, do sexo escolhido pela criança; 
b) Versão de Ayres e Reid (1996), na qual a pontuação é dividida em duas partes. Na primeira, a criança perde pontos para cada parte do corpo humano não desenhada e na segunda parte, ela ganha pontos para cada detalhe encontrado no desenho (ex: pupilas, sobrancelhas, roupas). O escore final é dado pela soma dos pontos, que é lançada em uma escala na qual -18 pontos equivale ao escore $0 ;-17$ ao escore $1 ; 0$ pontos equivale ao escore $18 \mathrm{e}$ assim sucessivamente, até atingir a pontuação máxima de +13 pontos, equivalente ao escore 31 . Quanto maiores a pontuação positiva e a pontuação final convertida, melhor o desempenho da criança no teste;

c) Procedimentos: Os testes utilizados fazem parte da rotina de acompanhamento do desenvolvimento infantil do ACRIAR. Os dados foram coletados por acadêmicas de terapia ocupacional, bolsistas de iniciação científica, treinados na aplicação dos testes, de acordo com as instruções contidas nos manuais específicos. Para garantir maior confiabilidade dos dados, os escores de todos os testes foram feitos por duas examinadoras independentes que, nos casos de discordância, reviam os resultados e decidiam, conjuntamente, qual a melhor pontuação para a criança. Todos os resultados foram revisados pela pesquisadora principal.

A aplicação dos testes foi individual, feita em local adequado, com mesa de tamanho apropriado para a criança e durou em média 40 minutos. Primeiramente, aplicou-se o MAC, sendo solicitado a criança que fizesse o traçado, sem sair da linha, no tempo determinado pela examinadora. Depois, as figuras do teste de Bender foram apresentadas para cópia e, por fim, aplicou-se o desenho da figura humana. Para este teste foi solicitado à criança que desenhasse um menino ou uma menina, deixando a escolha a seu critério. A pontuação de todos os testes seguiu as instruções especificadas nos manuais. Os pais das crianças foram esclarecidos dos objetivos da pesquisa, e todos assinaram termo de consentimento autorizando a participação da criança no estudo. $\mathrm{O}$ projeto foi aprovado pelo Comitê de Ética em Pesquisa - COEP/ UFMG.

Os dados foram analisados descritivamente e calculada a correlação entre as variáveis. Para se obter estimativa do nível de desempenho da criança em relação à idade, nos testes em que havia normas (AYRES, 1980; KROEFF, 1988; WECHSLER, 1996), foi feita a conversão do escore bruto médio para escore estandardizado. Como de regra, considerou-se que a média de desempenho do grupo ficou dentro do esperado, quando o escore estandardizado estava dentro do intervalo de \pm 1 desvio padrão da média específica para a idade do grupo. Para o teste de figura humana de Ayres e Reid (1966), como o estudo original reporta a média e desvio padrão para crianças típicas norte americanas de 6 e 7 anos, usou-se teste-t para examinar se o desempenho médio da amostragem em estudo era similar à média reportada para crianças de 6 anos. $\mathrm{O}$ exame da correlação entre os escores dos testes foi feito apenas com os escores brutos. A correlação de Pearson foi usada para dados com distribuição normal e a de Spearman para os dados que não se enquadraram nos padrões de normalidade.

\section{RESULTADOS}

$\mathrm{Na}$ Tabela 2, estão apresentadas as médias de desempenho em cada teste. Pode-se observar que as médias de desempenho do grupo de crianças ficaram dentro do esperado para a idade, em todos os testes, exceto o de Acuidade Motora ajustada (MAC-adj), o que é indicativo de maior lentidão do traçado, nas crianças pré-termo.

Tabela 2 - Média de escores e nível de desempenho nos testes.

\begin{tabular}{lccc}
\hline Teste & Escore bruto médio & Desvio padrão & Nível de desempenho para a idade \\
\hline F H Wechsler & 19,33 & 5,40 & na média \\
FH Ayres/Reid & 17,20 & 4,99 & na média* \\
MAC & 160,22 & 9,71 & na média \\
MAC ajustado & 139,55 & 10,57 & abaixo da média \\
Bender & 11,03 & 4,60 & na média \\
\hline
\end{tabular}

Nota: FH = Figura humana; MAC = Teste de acuidade motora escore bruto; MAC ajustado = Teste de acuidade motora - escore ajustado para tempo; Bender $=$ Teste Gestáltico de Bender. *Não foi observada diferença significativa entre as médias das crianças pré-termo brasileiras e crianças norte-americanas com desenvolvimento típico. 
A Tabela 3 mostra a correlação entre o peso e a idade gestacional ao nascimento e os escores dos testes aplicados. Observou-se correlação significativa entre o peso e a idade gestacional e entre a acuidade motora e o peso ao nascimento, indicando que, quanto menor o peso ao nascimento, menor a acuidade no traçado da criança. Correlações significativas, de moderada a alta, também foram encontradas entre os escores dos dois testes de desenho da figura humana e os dois escores do teste de acuidade motora.

Tabela 3 - Matriz de correlação entre as variáveis.

\begin{tabular}{|c|c|c|c|c|c|c|}
\hline & Idade gestacional & Peso ao nascimento & FH Wechsler & FH Ayres & MAC Acuidade & MAC ajustado \\
\hline Peso ao nascimento & $\mathbf{0 , 6 3 0} * *$ & & & & & \\
\hline FH Weschler & $-0,311$ & $-0,308$ & & & & \\
\hline FH Ayres & $-0,275$ & $-0,150$ & $0,761 * *$ & & & \\
\hline MAC - acuidade & $-0,251$ & $\mathbf{0 , 3 7 5 *}$ & $-0,082$ & 0,077 & & \\
\hline MAC ajustado & $-0,080$ & $0,412 *$ & 0,136 & 0,273 & $0,55 * *$ & \\
\hline Bender & $-0,066$ & 0,018 & $-0,230$ & $-0,192$ & $-0,19$ & $-0,237$ \\
\hline
\end{tabular}

Nota: $* * \mathrm{p}<0,01, * \mathrm{p}<0,05, \mathrm{FH}=$ Figura humana, MAC $=$ Acuidade motora.

\section{DISCUSSÃO}

Os resultados deste estudo, contrariamente às expectativas, não confirmam a relação entre a habilidade para desenhar a figura humana e a coordenação visomotora, em crianças com história de prematuridade. As crianças pré-termo examinadas apresentaram médias globais de desempenho, tanto nas duas versões do desenho da figura humana, como nos testes de coordenação visomotora, dentro do esperado para a idade. A única exceção foi o teste de acuidade motora, MAC ajustado, cujo resultado, abaixo do esperado para a idade, indica que, apesar de a acuidade motora estar dentro da média, as criança pré-termo são mais lentas para realizar o traçado.

Observa-se (Tabela 3) que a acuidade motora no traçado está correlacionada, significativamente, com o peso, mas não com a idade gestacional ao nascimento. A correlação positiva indica que, quanto menor o peso, pior a acuidade do traçado. Essa tendência vem sendo confirmada em estudos que incluem maior número de bebês com prematuridade extrema (GOYEN et al., 1998; GOYEN; LUI, 2002). Outro dado confirmado neste estudo, é que as crianças prétermo foram mais lentas no traçado do que o esperado para a idade. A observação das crianças durante a prova de traçado indica esforço aumentado e pausas freqüentes para descanso ou para reajustar a posição do lápis no papel. É importante ressaltar que, se na situação de teste as crianças tiveram tempo suficiente para fazer a prova em seu próprio ritmo, na escola, exigências tanto de acuidade como de velocidade, aumentam com a idade. Essa tendência a maior lentidão pode resultar em dificuldades para responder às demandas motoras na sala de aula, principalmente na escrita. Embora estudos longitudinais da criança prétermo sejam essenciais para confirmar essa dúvida, os dados aqui apresentados reforçam a posição de alguns autores de que é importante acompanhar a criança prétermo até a entrada no ensino fundamental (PIETZ et al., 2004).

Como era esperado, foi encontrada correlação significativa entre as duas versões de escore para o desenho da Figura Humana. Apesar de a versão de Ayres e Reid (1966) adotar uma perspectiva percepto-motora e a de Wechsler (1996) se caracterizar como avaliação cognitiva, deve-se lembrar que ambas partiram do trabalho original de Goodenough. A correlação é alta $(\mathrm{r}=0,76)$, mas não atinge o valor de 0,90 , que indicaria coincidência de resultados, ou seja, os dois sistemas de escore têm pontos em comum, porém, não são equivalentes. Apesar do cunho perceptual de uma das versões, nenhuma delas apresentou correlação significativa com os testes visomotores aplicados. No 
estudo de Ayres e Reid (1966), foi observada correlação significativa entre o desenho da Figura humana e provas perceptuais, incluindo o MAC e a cópia de figuras com papel e lápis, mas, principalmente, com testes de percepção de posição no espaço e relações espaciais. Os autores sugerem que esta versão do teste do desenho da Figura humana seria mais efetiva como indicador da percepção visual (AYRES; REID, 1966).

Para nossa surpresa, também não foram encontradas correlações significativas entre os outros testes percepto-motores aplicados. Esses dados nos levam a considerar que os testes usados, apesar de serem considerados testes visomotores, possivelmente avaliam diferentes aspectos da percepção visual e coordenação visomotora. O teste de Bender (KOPPITZ, 1989), por envolver cópia de figuras, tem elementos espaciais; já o teste de acuidade motora (AYRES, 1984) é puramente motor, pois exige apenas traçado sobre uma linha. A habilidade para desenhar a Figura humana, por sua vez, é influenciada são só pela coordenação visomotora, mas também pelas experiências motoras anteriores da criança, pelo conceito de partes do corpo, além das contribuições da escolaridade e do ambiente sociocultural (BANDEIRA et al., 1994). Ou seja, os testes usados nesse estudo têm similaridades, mas avaliam aspectos perceptuais e motores diferenciados. Fica evidente que a avaliação das habilidades perceptuais e motoras é um processo complexo, que exige mais de um instrumento e definição criteriosa do que exatamente se deseja avaliar.

Uma outra possibilidade é que, considerando que as provas usadas nesse estudo dependem, pelo menos em parte, de experiência previa, era esperado que crianças pré-termo, provenientes de famílias de baixa renda, algumas inclusive sem experiência escolar, tivessem pior desempenho nos testes. Como isso não ocorreu, levantamos a hipótese de que os critérios e normas de escore dos testes possam estar desatualizados ou lenientes para a amostragem, pois, em sua maioria, foram padronizados há mais de duas décadas. O teste do desenho da Figura Humana de Wechsler (1996) apesar de mais recente, já tem nova versão em andamento, com amostragem mais ampla, que não estava disponível no início do estudo. Critérios lenientes resultam em pontuações mais altas e, como conseqüência, menor variabilidade nos dados. Como se sabe, correlações tendem a ser mais baixas se há maior homogeneidade nos dados (HINKLE et al., 1988). Tais resultados nos levam a questionar a validade dos testes aqui usados, para detectar alterações sutis no desempenho perceptual-motor das crianças avaliadas.

\section{CONCLUSÃO/RECOMENDAÇÕES}

Embora os dados apresentados não nos permitam tirar conclusões definitivas, os resultados mostram que existe correlação entre peso ao nascimento e acuidade do traçado, sendo que crianças nascidas com menos de 34 semanas de idade gestacional, aos seis anos de idade, tendem a ser mais lentas no traçado do que o esperado para a idade. Como essa lentidão pode ter implicações para o desempenho escolar, é importante dar seguimento a estudos nessa área e, como medida preventiva, recomendamos que se dê mais atenção à escolaridade de crianças com história de prematuridade, como descrito nesse estudo.

A correlação entre as duas versões do teste do desenho da Figura humana foi alta, indicando que os dois sistemas de pontuação tem muitos pontos em comum. Apesar da versão de Wechsler (1996) ser padronizada para crianças brasileira, por se tratar de teste cognitivo, é de uso exclusivo de psicólogos, sendo usada nesse estudo, exclusivamente para fins de pesquisa, com suporte da equipe interdisciplinar do ACRIAR. Como a versão de Ayres e Reid (1966) foi desenvolvida por terapeuta ocupacional, ela pode ser usada, em associação com outros testes perceptomotores, para avaliar o desenvolvimento da criança nessa área. É importante, no entanto, contarmos com normas de desempenho para crianças brasileiras.

O fato de a maioria das crianças ter obtido desempenho dentro do esperado para a idade, nos testes percepto-motores usados nesse estudo, sugere que crianças pré-termo não apresentam problemas nessa área. Esse resultado, no entanto, não está de acordo com a literatura internacional (GOYEN et al., 1998; LIEBHARDT et al., 2000; PIETZ et al., 2004). Uma hipótese alternativa é que os testes usados apresentam problema de validade para a amostragem em questão, o que nos leva a refletir sobre um antigo dilema: face à carência de instrumentos brasileiros atualizados, pesquisadores acabam usando testes importados, cujos resultados são de difícil interpretação, como no presente estudo. Não é objetivo deste trabalho discutir essa questão, mas considera-se essencial o esforço para o desenvolvimento de testes mais adequados à realidade brasileira e ao contexto de atuação dos terapeutas ocupacionais. 
CARVALHO, D. J.; MAGALHÃES, L. C. The relationship between human figure drawing and visual-motor coordination in 6-year-old preterm children. Rev. Ter. Ocup. Univ. São Paulo, v. 15, n. 3, p. 98-105, set./dez., 2004.

\begin{abstract}
S: The objective of this study was to examine the performance of preschool age children born prematurely in perceptual and visomotor tests. As prematurity has an impact on child's development it is important to evaluate the percepto-motor skills in this population. The participants of this study were 30 children, 20 girls and 10 boys, born with gestational age $\geq 34$ weeks, recruited in a developmental follow-up program for preterm infants. The children were assessed with the Motor Accuracy test (AYRES, 1980), the Bender Gestalt (KOPPITZ, 1989) and the versions of the human figure drawing by Wechsler (1996) and by Ayres and Reid (1966). The results showed that the preterm children had age appropriated performance in all tests but the motor accuracy adjusted for time, which indicates that children born pre-term present are slower in tracing. There was a significant correlation between the two versions of the human figure drawing (Pearson $r=0,76$ ) and between birth weight and motor accuracy (Pearson $r=0,36$ e 0,41 ), moreover, none of the other correlations between the test were significant. These results are in contrast with the literature, which indicates that preterm children present lower performance in perceptual and motor tests. The poor correlation among the scores of the different tests and the signs of poor clinical utility lead to doubts regarding the validity of the instruments utilized in the study. It was concluded that there is a need to develop instruments with current norms for the Brazilian children, that will allow for more accuracy in test results.
\end{abstract}

KEY WORDS: Infant, premature. Psychomotor performance. Motor skills disorders.

Agradecemos ao CNPq e à FAPEMIG pelo auxílio financeiro e pela concessão de bolsas; à equipe do ACRIAR, pelo suporte ao trabalho interdisciplinar e, principalmente, às crianças e familiares que tornaram possível a condução deste estudo.

\title{
REFERÊNCIAS
}

\author{
AYRES, A. J. Southern California sensory integration \\ tests. Los Angeles, LA: Western Psychological, 1980.
}

AYRES, A. J.; REID, W. The self-drawing as an expression of perceptual-motor dysfunction. Cortex, v. 2, p. 254-265, 1966.

BANDEIRA, D. R.; HUTZ, C. S. A contribuição dos testes DFH, Bender e Raven na predição do rendimento escolar na primeira série. Psicol. Teor. Pesqui., Brasília, v. 10, n.1, p. 59-72, 1994.

BRITTAIN, W. L.; CHIEN, Y. Relationship between preschool children's ability to name body parts and their ability to construct a man. Percept. Mot. Skills, v. 57, p. 19-24, 1983.

CARVALHO, A. E. V.; LINHARES, M. B. M.; MARTINEZ, F. E. História de desenvolvimento e comportamento de crianças nascidas pré-termo e baixo-peso $(<1500 \mathrm{~g})$. Psicol. Reflex. Crit., v. 14, n. 1, p. 1-33, 2001.

GOODENOUGH, F. L. Measurement of intelligence by drawings. Chicago, Il: World Book Company, 1926 apud AYRES, A. J.; REID, W. The self-drawing as an expression of perceptual-motor dysfunction. Cortex, v. 2, p. 254-265, 1966.
GOYEN, T. A.; LUI, K.; WOODS, T. Visual-motor, visualperceptual, and fine motor outcomes in very-low-birthweight children at 5 years. Dev. Med. Child Neurol., v. 40, p. 7081, 1998.

GOYEN, T. A.; LUI, K. Longitudinal motor development of "apparently normal" high-risk infants at 18 months, 3 and 5 years. Early Hum. Dev., v. 70, p. 10-4, 2002.

GROOT, L. Posture and motility in preterm infants. Dev. Med. Child Neurol., v. 42, p. 65-8, 2000.

HINKLE, D. E.; WIERSMA, W.; JURS, S. G. Applied statistics for the behavioral sciences. Boston: Houghton Mifflin Co, 1988.

KEPHART, N. C. O aluno de aprendizagem lenta. 2. ed. Porto Alegre: Artes Médicas, 1990.

KOPPITZ E. O teste gestáltico de Bender para crianças. 2. ed. Porto Alegre: Artes Médicas, 1989.

KROEFF, P. Normas brasileiras para o teste de Bender. Psicol. Reflex. Crit., v. 3, p. 10-7, 1988. 
LIEBHARDT, G.; SONTHEIMER, D.; LINDERKAMP, O. Visual-motor function of very low birth weight and full-term children at 3 to 4 years of age. Early Hum. Dev., v. 57, n. 1, p. 33-47, 2000.

LUOMA, L.; HERRGARD, E.; MARTIKAINEM, A. Neuropsychological analyses of the visuo motor problems in children born preterm at $<32$ weeks of gestation a 5 -years prospective follow-up. Dev. Med. Child Neurol., v. 40, p. 21-30, 1998

MAGALHÃES, L. C.; BARBOSA, V. M.; LOPES, K. C.; PAIXÃO, M. L. Estudo longitudinal do desenvolvimento do recém-nascido pré-termo: avaliação na idade pré-escolar. Rev. Bras. Neurol., v. 35, n. 4, p. 87-93, 1999.

MAGALHÃES, L. C.; WENDLING, P. C.; PAIXÃO, M. L.; MANCINI, M. C.; BARBOSA, V. M. Estudo comparativo sobre o desempenho perceptual e motor na idade escolar em crianças nascidas pré-temo e a termo. Arq. Neuropsiquiatr., v. 61, p. $250-5,2003$.

MELO, J. P. Desenvolvimento da consciência corporal. Uma experiência da educação física na idade pré-escolar. Campinas: UNICAMP, 1997.

OAKLAND, T.; DOWLING, L. The draw-a-person test: validity properties for nonbiased assessment. Learn. Disabil. Q., v. 6, 1983

PIETZ, J.; PETER, J.; GRAF, R.; RAUTERBERGRULAND, I.; RUPP, A.; SONTHEIMER, D.; LINDERKAMP, O. Physical growth and neurodevelopmental outcome of nonhandicapped low-risk children born preterm. Early Hum. Dev., v. 79, n. 2, p. 131-43, 2004.

SHOT-DEGRAFF, M. A.; SLANSKY, K. E. Validity of preschoolers' self-drawings as an index of human figure drawing performance. Occup. Ther. J. Res., v .9, n. 5, p. 305-14, 1989.

SCPE - Surveillance of cerebral palsy in Europe: a collaboration of cerebral palsy surveys and registers. Dev. Med. Child Neurol., v. 42, p. 816-24, 2000.

WECHSLER, S. M. O desenho da figura humana: avaliação do desenvolvimento cognitivo infantil. Manual para crianças brasileiras. Campinas: Psy, 1996.

WOLKE, D; MEYER, R. Cognitive status, language attainment and pre-reading skills of 6-year-old preterm children and their peers: the Bavarian Longitudinal Study. Dev. Med. Child Neurol., v. 41, p. 94-109, 1999. 